\title{
Integrated mRNA and small RNA sequencing reveals microRNA regulatory network associated with internode elongation in sugarcane (Saccharum officinarum L.)
}

Lihang Qiu ${ }^{1,2+}$, Rongfa Chen ${ }^{1,2+}$, Yegeng Fan ${ }^{1,2}$, Xing Huang ${ }^{1,2}$, Hanmin Luo ${ }^{1,2}$, Faqian Xiong ${ }^{2}$, Junxian Liu ${ }^{1,2}$, Ronghua Zhang ${ }^{1,2}$, Jingchao Lei ${ }^{1,2}$, Huiwen Zhou ${ }^{1,2}$, Jianming $\mathrm{Wu}^{1,2^{*}}$ and Yangrui $\mathrm{Li}^{1,2^{*}}$ (D)

\begin{abstract}
Background: Internode elongation is one of the most important traits in sugarcane because of its relation to crop productivity. Understanding the microRNA (miRNA) and mRNA expression profiles related to sugarcane internode elongation would help develop molecular improvement strategies but they are not yet well-investigated. To identify genes and miRNAs involved in internode elongation, the CDNA and small RNA libraries from the preelongation stage (EI), early elongation stage (EII) and rapid elongation stage (EIII) were sequenced and their expression were studied.

Results: Based on the sequencing results, 499,495,518 reads and 80,745 unigenes were identified from stem internodes of sugarcane. The comparisons of El vs. Ell, El vs. Elll, and Ell vs. Elll identified 493, 5035 and 3041 differentially expressed genes, respectively. Further analysis revealed that the differentially expressed genes were enriched in the GO terms oxidoreductase activity and tetrapyrrole binding. KEGG pathway annotation showed significant enrichment in "zeatin biosynthesis", "nitrogen metabolism" and "plant hormone signal transduction", which might be participating in internode elongation. miRNA identification showed 241 known miRNAs and 245 novel candidate miRNAs. By pairwise comparison, 11, 42 and 26 differentially expressed miRNAs were identified from El and EII, El and EIII, and EII and EIII comparisons, respectively. The target prediction revealed that the genes involved in "zeatin biosynthesis", "nitrogen metabolism" and "plant hormone signal transduction" pathways are targets of the miRNAs. We found that the known miRNAs miR2592-y, miR1520-x, miR390-x, miR5658-x, miR6169-x and miR8154-x were likely regulators of genes with internode elongation in sugarcane.

Conclusions: The results of this study provided a global view of mRNA and miRNA regulation during sugarcane internode elongation. A genetic network of miRNA-mRNA was identified with miRNA-mediated gene expression as a mechanism in sugarcane internode elongation. Such evidence will be valuable for further investigations of the molecular regulatory mechanisms underpinning sugarcane growth and development.
\end{abstract}

Keywords: Transcriptome, Next-generation sequencing, Zeatin biosynthesis, Nitrogen metabolism, Plant hormone signal transduction

\footnotetext{
*Correspondence: wujianming2004@126.com; liyr5745@126.com

†Lihang Qiu and Rongfa Chen contributed equally to this work.

${ }^{1}$ Sugarcane Research Institute, Guangxi Academy of Agricultural Sciences/

Sugarcane Research Center, Chinese Academy of Agricultural Sciences, East

Daxue Road 172, Nanning 530004, Guangxi, China

Full list of author information is available at the end of the article
}

(c) The Author(s). 2019 Open Access This article is distributed under the terms of the Creative Commons Attribution 4.0 International License (http://creativecommons.org/licenses/by/4.0/), which permits unrestricted use, distribution, and

reproduction in any medium, provided you give appropriate credit to the original author(s) and the source, provide a link to the Creative Commons license, and indicate if changes were made. The Creative Commons Public Domain Dedication waiver (http://creativecommons.org/publicdomain/zero/1.0/) applies to the data made available in this article, unless otherwise stated. 


\section{Background}

Internode elongation is a major feature that affects plant growth, errectness, biomass and ultimately yield [1]. Thus, the genetics and the regulatory mechanisms of internode elongation in crop plants have been extensively investigated. Genetic and environmental factors such as gene expression [2-4], genomic variation $[5,6]$, hormonal regulation $[7,8]$, nutrients $[9,10]$, light [11], water [12] and temperature [13] control internode elongation. Of these regulatory factors, hormonal manipulation is an effective and efficient approach to promote crop growth to promote productivity [14-16].

Complex hormonal mechanisms are associated with internode elongation. For example, auxin [14], gibberellin [17] and brassinosteroids [18] induce internode elongation. In contrast, abscisic acid [19], ethylene [20] and jasmonic acid [21] suppress internode elongation in plants. Further, different species and growing condition add additional complexity to growth and developmental regulation. Understanding sugarcane crop-specific mechanisms of hormonal regulation stem growth would help improve crop productivity. Alternatively, endogenous hormones can be manipulated by genome editing [22, 23] and RNA interference technologies [24, 25]. microRNAs (miRNAs), being an effective RNA interference mechanisms, show the prospect of regulating hormone production and action [26-28]. TIR1 and AFB, part of auxin signaling, are targets of miR393, and the suppressive effects of miR393 on auxin are indicated in Arabidopsis [29]. GAMYB, a gene in gibberellin signal pathway, is regulated by miR159 [30]. Also, hormones regulate miRNA expression in plants. For instance, with deep sequencing of abscisic acid-treated tomato (Solanum lycopersicum), 269 differentially expressed miRNAs were identified [31].

Development of sequencing technology has facilitated transcriptome studies that provide unprecedented detail about the molecular biological processes in plants [32, 33]. Transcriptome sequencing approaches promise increased understanding of the expression patterns and molecular regulatory mechanisms in gene expression [34]. By transcriptome sequencing, the genes associated with culm elongation in bamboo (Dendrocalamus sinicus) were identified [35]. In another study, transcriptome sequencing showed the changes in gene expression via induction of ethephon in maize (Zea mays) plants [36]. These studies provide basic information about the functional genes involved in internode elongation. In cotton (Gossypium hirsutum), 64 differentially expressed miRNAs were identified during the fiber elongation process [37]. The miRNA profiles during tissue differentiation and growth revealed by small RNA sequencing may provide new insight for epigenetic regulation, which might determine a starting point toward important questions regarding plant growth.
Sugarcane (Saccharum officinarum L.) is an economically important crop that is widely planted in tropical and subtropical regions [38]. Sugarcane is used for producing ethanol and raw sugar; thus, this valuable crop is grown around the world [39]. Understanding the genetic control of sugarcane growth, particularly the biological process of internode elongation, would accelerate the industrial development of sugarcane cultivation. Investigation of miRNA-mRNA networks in sugarcane could reinforce further crop gains. Although several studies demonstrate changes in gene expression or miRNAs during internode elongation [40,41], the present study focused on the integrated analysis of miRNA and mRNA interactions, which should produce an image of the mRNA-miRNA networks that occur between transcriptional and posttranscriptional regulation in this biological process.

To better understand the molecular changes and regulation of gene expression by miRNA, we sequenced mRNA and small RNA libraries from internode tissues at different stages including the pre-elongation stage, early elongation stage and rapid elongation stage. The libraries from these tissues were sequenced by an Illumina Hiseq 4000 platform. By comparing the differential expression, the candidate genes and miRNAs involved in internode elongation were identified. Furthermore, the mRNA and miRNA interaction network was built by target prediction using a bioinformatic approach. These integrated mRNA and small RNA sequencing results provide pioneering evidence for a view of candidate internode elongation-associated miRNAs in sugarcane and may be useful for development of potential functional markers to develop molecular breeding.

\section{Results \\ mRNA expression profiles in different stages from internodes}

To understand the molecular mechanism of internode elongation in sugarcane, nine cDNA libraries from the pre-elongation stage (EI), early elongation stage (EII) and rapid elongation stage (EIII) were sequenced. Three biological replicates (individuals) from the stages were included from which a total of 499,495,518 reads were obtained. After filtering, 484,324,322 clean reads were identified (Table 1). All the clean reads were used to perform de novo assembly, which generated 80,745 unigenes. The average length of unigenes was $900 \mathrm{bp}$, and N50 was 1600 bp.

The gene expression in the present study was calculated by the RPKM method. Hierarchical clustering of all the unigenes showed that the biological replicates from each stage were separately clustered together (Fig. 1a). After normalizing the gene expression of all the unigenes, the results of principal component analysis showed a distinct 
Table 1 Summary of the RNA-Seq Data

\begin{tabular}{|c|c|c|c|c|c|}
\hline Sample & Before Filter Read Number & After Filter Read Number & GC (\%) & Q20 (\%) & Q30 (\%) \\
\hline El-1 & $50,744,342$ & $49,382,978$ & $55.90 \%$ & $95.85 \%$ & $90.31 \%$ \\
\hline $\mathrm{El}-2$ & $47,767,534$ & $46,374,160$ & $56.12 \%$ & $95.70 \%$ & $90.09 \%$ \\
\hline $\mathrm{El}-3$ & $63,212,086$ & $61,335,446$ & $55.75 \%$ & $95.68 \%$ & $90.07 \%$ \\
\hline Ell-1 & $46,876,844$ & $45,390,562$ & $56.09 \%$ & $95.56 \%$ & $89.86 \%$ \\
\hline Ell-2 & $60,658,428$ & $58,791,718$ & $56.70 \%$ & $95.61 \%$ & $89.94 \%$ \\
\hline Ell-3 & $68,915,150$ & $66,614,358$ & $61.35 \%$ & $95.29 \%$ & $89.27 \%$ \\
\hline EIII-1 & $53,018,772$ & $51,122,918$ & $55.71 \%$ & $95.28 \%$ & $89.35 \%$ \\
\hline EIII-2 & $54,777,846$ & $53,228,728$ & $56.98 \%$ & $95.61 \%$ & $89.71 \%$ \\
\hline EIII-3 & $53,524,516$ & $52,083,454$ & $55.99 \%$ & $95.82 \%$ & $90.26 \%$ \\
\hline
\end{tabular}

position of the EI, EII and EIII stages, indicating significant changes among the stages in the transcriptome (Additional file 1). There was greater separation on PC1 for samples from the EII groups, indicating a stronger transcriptional differentiation during early elongation stage (Fig. 1b).

\section{Differentially expressed genes in different stages of internode development}

To compare the gene expression in different stages of internode elongation, the RSEM package was used to identify differentially expressed genes. In the comparisons between EI and EII, EI and EIII, and EII and EIII, 493, 5035 and 3041 differentially expressed genes were identified, respectively (Fig. 2a). Between the EI and EII stages, 234 genes were up-regulated and 259 genes were down-regulated. The most differentially expressed genes were in the comparison between EI and EIII stages, which included 1601 up-regulated and 3434 downregulated genes at EIII stage. 1,061 and 1,980 genes increased and decreased at EII stage, respectively, by compared with EII and EIII stages (Fig. 2b). The Venn plot showed that 17 differentially expressed genes overlapped among the three comparisons (Fig. 2b, Table 2). The overlap between EI vs. EIII and EII vs. EIII had the maximum number of genes (1,483 differentially expressed genes), whereas the overlap of EI vs. EII and EI vs. EIII had the fewest number of genes (221 differentially expressed genes). The overlap between EI vs. EII and EII vs. EIII had 226 differentially expressed genes (Fig. 2c, Additional file 1).

\section{Functional annotation of the differentially expressed genes}

To reveal the function of differentially expressed genes in different stages from internodes, GO and KEGG enrichment analyses were performed. Only 3 GO terms were enriched (q-value $<0.05$ ) in the comparison EI vs. EII: two oxidoreductase activity terms and tetrapyrrole binding. It was found that $11 \mathrm{GO}$ terms were enriched (q-value $<0.05$ ) in the EI vs. EIII comparison, which included DNA binding, carboxypeptidase activity, hydrolase activity, oxidoreductase activity, nitrate reductase activity, nucleic acid binding transcription factor activity, tetrapyrrole binding, transmembrane transporter activity, and transporter activity. The comparison between EII and EIII indicated that $29 \mathrm{GO}$ terms were enriched (Fig. 3a, Additional file 2).

From the KEGG enrichment results, it was found that 16 KEGG pathways were enriched. From the EI vs. EII comparison, 9 enriched pathways were identified, including "zeatin biosynthesis" and "nitrogen metabolism", which involved tissue growth. The EI vs. EIII comparison contained 11 enriched pathways, including "plant hormone signal transduction" and "nitrogen metabolism" associated with growth. For EII vs. EIII, 7 enriched pathways were found, including "nitrogen metabolism" (Fig. 3b, Additional file 3).

The expression profiles of ten candidate genes from "zeatin biosynthesis", "nitrogen metabolism" and "plant hormone signal transduction" pathways were investigated by qPCR. The expression profiles by qPCR were similar to the results of transcriptome analysis (Fig. 4).

\section{Sequencing of small RNAs in internodes}

The sequencing of small RNAs was investigated to unveil the dynamic regulation of miRNAs on gene expression during internode elongation in sugarcane. Nine small RNA libraries were sequenced, and a total of 137 , 610,370 clean reads (Table 3 ) were generated. The length distribution showed that most of these reads were in the range of miRNA from 18 to $24 \mathrm{nt}$. After removing the rRNA, snRNA, snoRNA, and tRNA by BLAST against the GenBank and Rfam databases, the remaining small RNAs were retained for the following analysis.

\section{Identification of known and novel miRNAs}

The known miRNAs were conducted by blastn to hit the miRBase. A total of 241 known miRNAs were detected in the internode tissues from sugarcane. From all the 


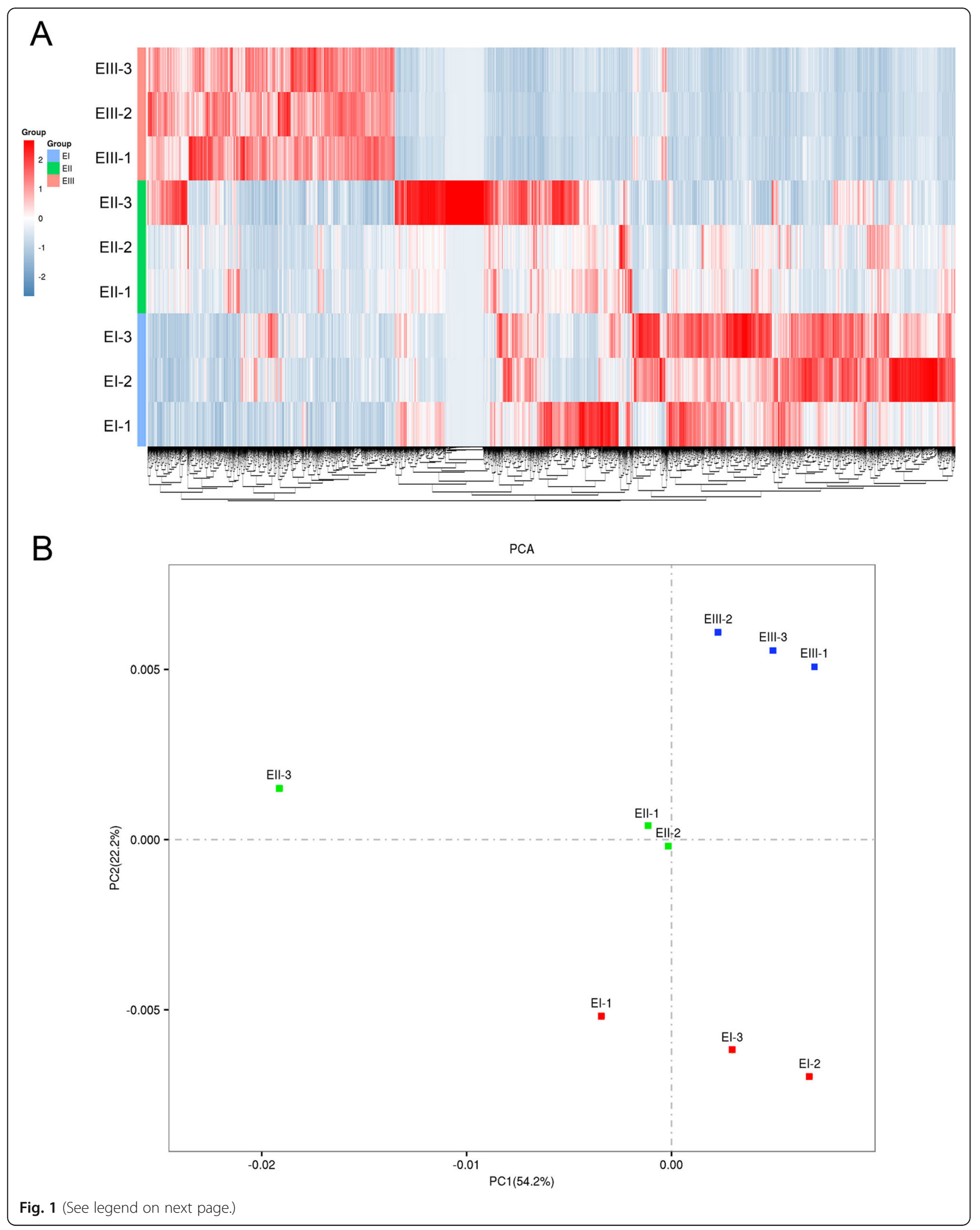


(See figure on previous page.)

Fig. 1 Overview of mRNA Expression Profiles at Different Stages of Internode Elongation in Sugarcane. a Heat map comparison of the preelongation stage (EI), early elongation stage (EII) and rapid elongation stage (EIII). The Z-score calculated from RPKM values for each gene were used. Gene expression is colored for low (blue) to high (red). Each line represents a single gene and each row shows a library listed on the left. $\mathbf{b}$ Two-component principal component analysis of the nine transcriptomes from the pre-elongation stage (El), early elongation stage (EII) and rapid elongation stage (EIII). The percentages represent the variances captured by the principal component 1 (PC1) and principal component 2 (PC2).

Red, blue and green dots indicate El, Ell and Elll, respectively

sequenced libraries, 118 known miRNAs were overlapped in all the groups. miR168-x, miR319-y, miR168-y, miR396- $x$ and miR166-y were the most abundant known miRNAs. The novel miRNAs were predicted by the mireap v0.2 package. A total of 245 novel candidate miRNAs were found in the internodes from sugarcane (Table 3, Additional file 4). Novel-miR0183-5p, novelmiR0209-5p and novel-miR0183-3p were the most abundant novel miRNAs.

\section{Differentially expressed miRNAs and their targets}

To understand the miRNA regulatory mechanism in internode elongation, the differentially expressed miRNAs were identified by the TPM method. In the comparisons between EI and EII, EI and EIII, and EII and EIII, 11, 42 and 26 differentially expressed miRNAs were found, respectively. Between stages EI and EII, 4 up regulated and 7 down regulated miRNAs were found. The most extensive differentially expressed miRNAs were found between stages EI and EIII, with 12 and 30 that were up-regulated and down-regulated, respectively. Finally, 5 up-regulated and 21 down-regulated miRNAs were found in the comparison EII vs. EIII (Fig. 5a). In the EII vs. EIII comparison, 14 miRNAs with their 75 targets were identified. The principal component analysis indicated a distinct position of the EI, EII and EIII stages (Fig. 5b, Additional file 5).

Targets of the differentially expressed miRNAs were detected. For 8 of the total differentially expressed miRNAs in the EI and EII comparison, the target unigenes were identified (78 in total), whereas no targets were identified for the other 3 miRNAs. In the EI and EIII comparison, 204 targets for 31 miRNAs were identified (Additional file 6).
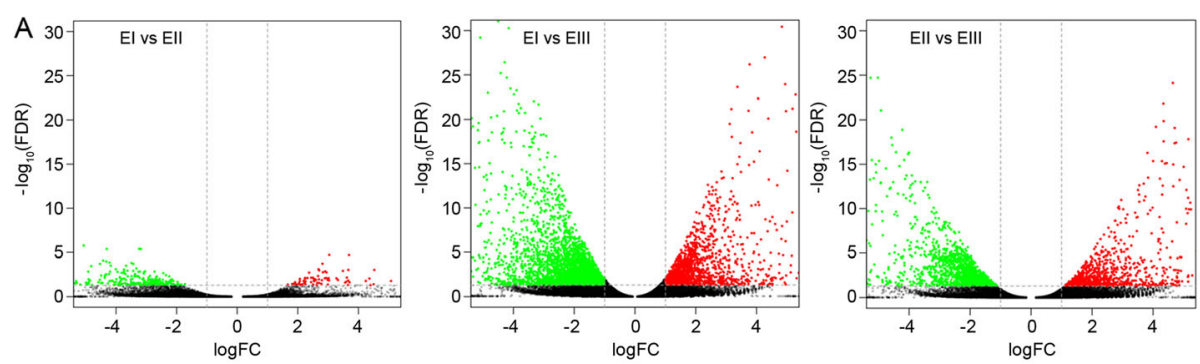

C

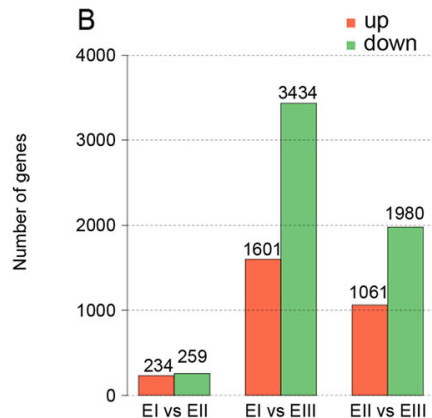

Fig. 2 Differential Expression of Genes in Different Stages of Internode Elongation in Sugarcane. a Volcano plot shows the differentially expressed genes (green dot: down-regulated genes, red dot: up-regulated genes, black dot: unchanged genes). Each dot represents a single gene. Significant down-regulated genes and up-regulated genes are identified with $|\log 2 \mathrm{FC}| \geq 1$ and FDR cutoff< 0.05 . $X$ axis shows $\log 2 \mathrm{FC}$ value and $Y$ axis shows $-\log _{10}$ (FDR). b Number of differentially expressed genes in the comparisons of El vs. Ell, El vs. Elll, and Ell vs. Elll. Red bars represent the number of up-regulated genes. Green bars represent the number of down-regulated genes. The number on each bar indicates the count of up-regulated or down-regulated genes. c Venn diagram shows overlap of differentially expressed genes among the comparisons. The red, purple and green circle represents comparisons of El vs. Ell, El vs. Elll, and Ell vs. Elll, respectively 
Table 2 Summary of Transcriptome Assembly Statistics

\begin{tabular}{lcccccc}
\hline Gene Number & GC (\%) & N50 (bp) & Max. length (bp) & Min. length (bp) & Average length (bp) & Total assembled bases (bp) \\
\hline 80,745 & 50.50 & 1600 & 14,063 & 201 & 900 & $72,689,277$ \\
\hline
\end{tabular}

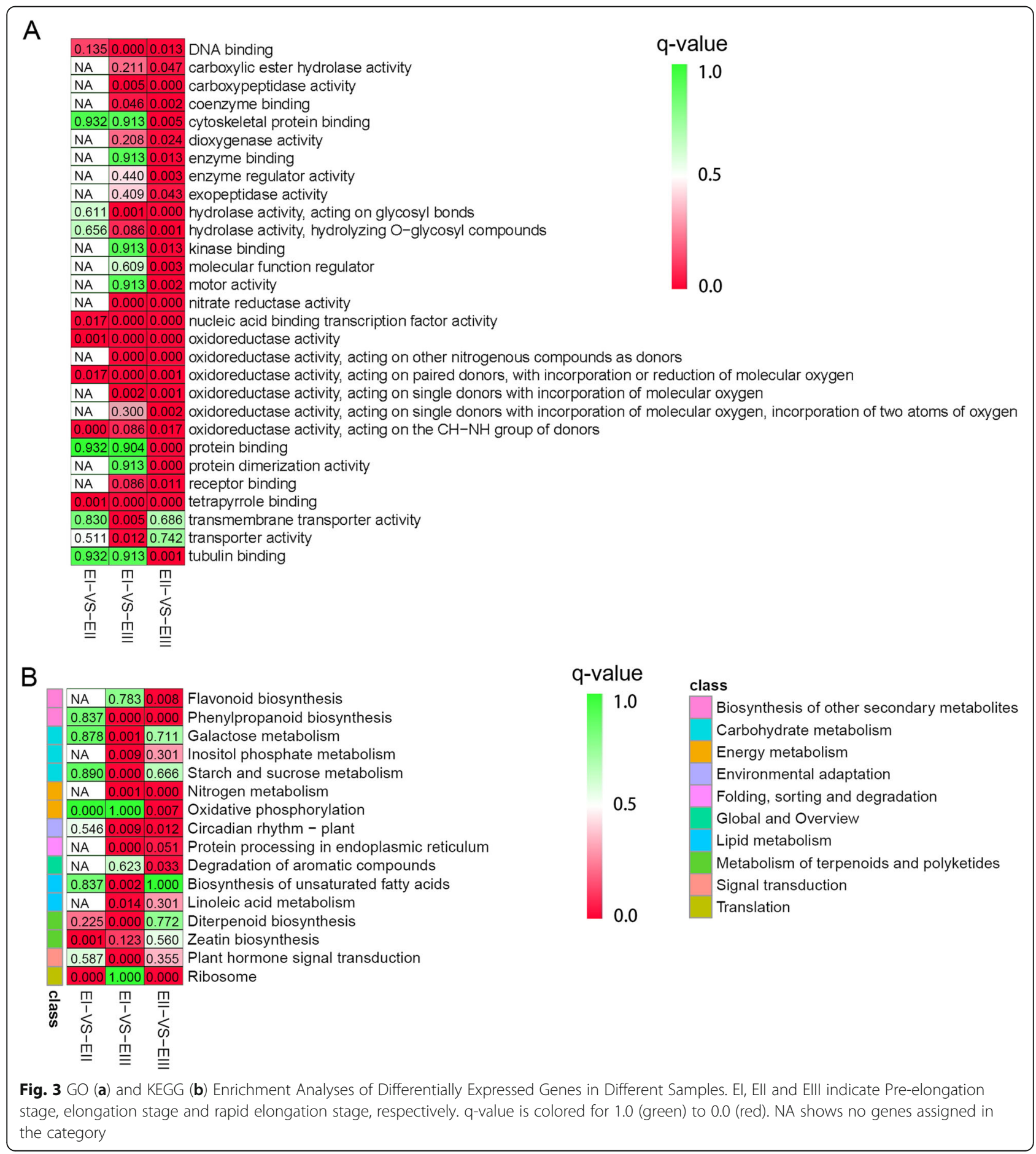




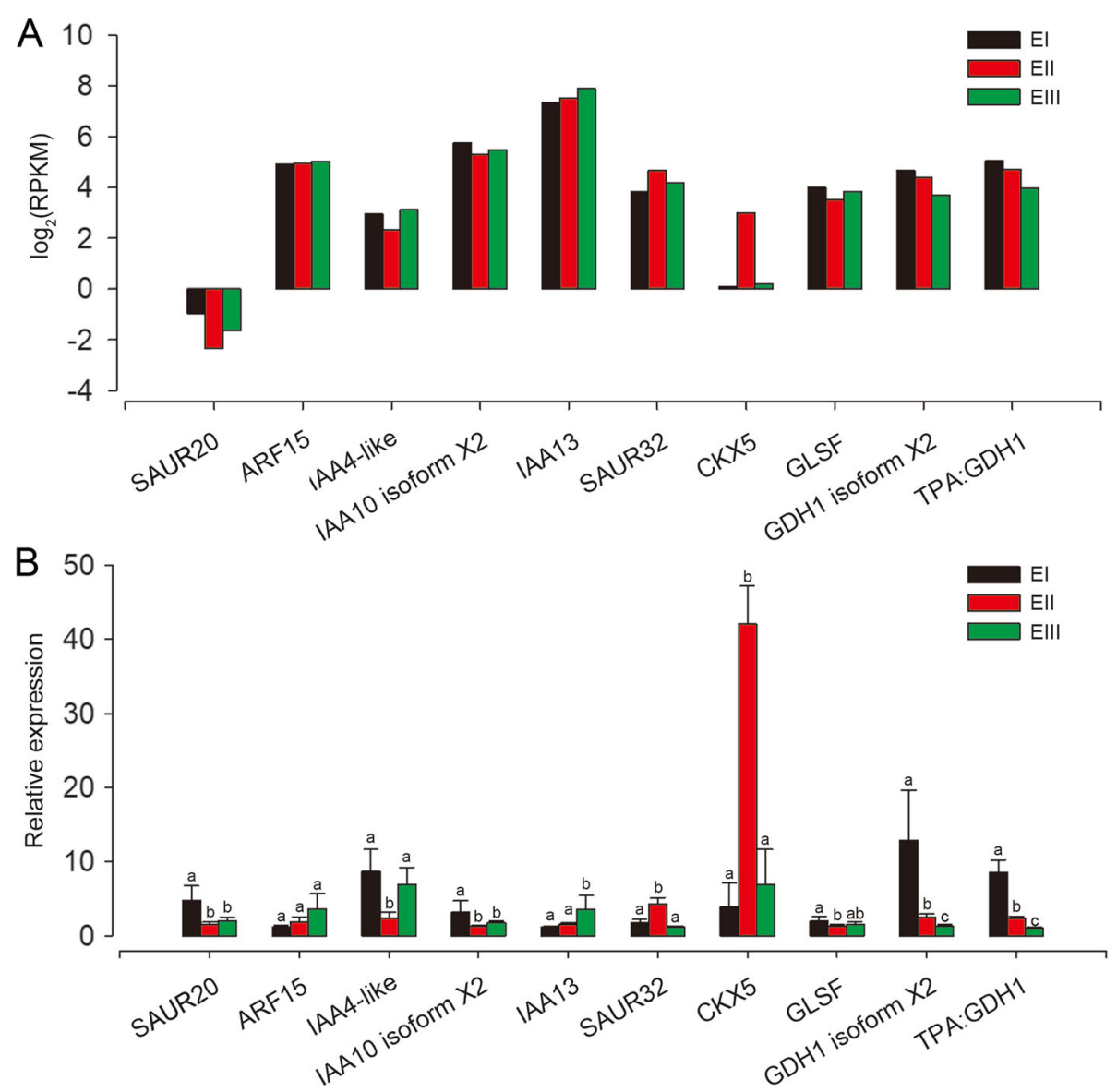

Fig. 4 Expression Levels of Ten Candidate Genes in different stages. a Expression of 10 candidate genes from RNA-seq calculated by the RPKM method using the RSEM package. The expression is shown as $\log _{2}$ (RPKM). $\mathbf{b}$ Expression of 10 candidate genes from qPCR. The gPCR results are represented at each stage as the mean \pm SD. The significant differences $(P<0.05)$ among the PCR results in different groups were indicated using different letters on each bar. El (black bars), Ell (red bars) and Elll (green bars) indicate pre-elongation stage, elongation stage and rapid elongation stage, respectively

Differentially expressed mRNA and miRNA pairs related to internode elongation

Internode elongation is a tissue growth process, and therefore, the mRNA and miRNA network related to tissue growth were identified. As found in the present analysis,

Table 3 Summary of Small RNA Data and miRNA Annotation

\begin{tabular}{llll}
\hline Sample & Read Number & Known miRNA number & Novel miRNA number \\
\hline El-1 & $15,140,390$ & 145 & 149 \\
El-2 & $16,845,194$ & 152 & 172 \\
El-3 & $15,786,890$ & 155 & 152 \\
Ell-1 & $15,759,580$ & 152 & 164 \\
Ell-2 & $13,794,648$ & 152 & 183 \\
EII-3 & $14,105,373$ & 156 & 162 \\
EIII-1 & $14,745,034$ & 157 & 175 \\
EIII-2 & $14,864,950$ & 162 & 170 \\
EIII-3 & $16,568,311$ & 178 & 194 \\
\hline
\end{tabular}

"zeatin biosynthesis", "nitrogen metabolism" and "plant hormone signal transduction" pathways were involved in internode elongation. For identification of mRNAs and their corresponding miRNAs in these pathways, 2, 3 and 37 pairs of miRNA-mRNA were found from "zeatin biosynthesis", "nitrogen metabolism" and "plant hormone signal transduction" pathways, respectively (Additional file 7). Negative correlations (rho between -0.87 and -1) between these miRNA and mRNA pairs were observed. The expression of these genes and miRNAs was shown in Fig. 6. In the "zeatin biosynthesis" pathway, novel-m0140-5p and novel-m01393p targeted cytokinin dehydrogenase 5 precursor (CKX5) and cytokinin hydroxylase-like isoform X1 (CYP735A1), respectively. miR2592-y targeted glutamate dehydrogenase (GDH1) isoform X2 and TPA, and glutamic dehydrogenase1 (GDH1) and novel-m0204-5p targeted ferredoxindependent glutamate synthase (GLSF), the chloroplastic precursor in "nitrogen metabolism" pathways. "Plant hormone signal transduction" had the most miRNA and mRNA 

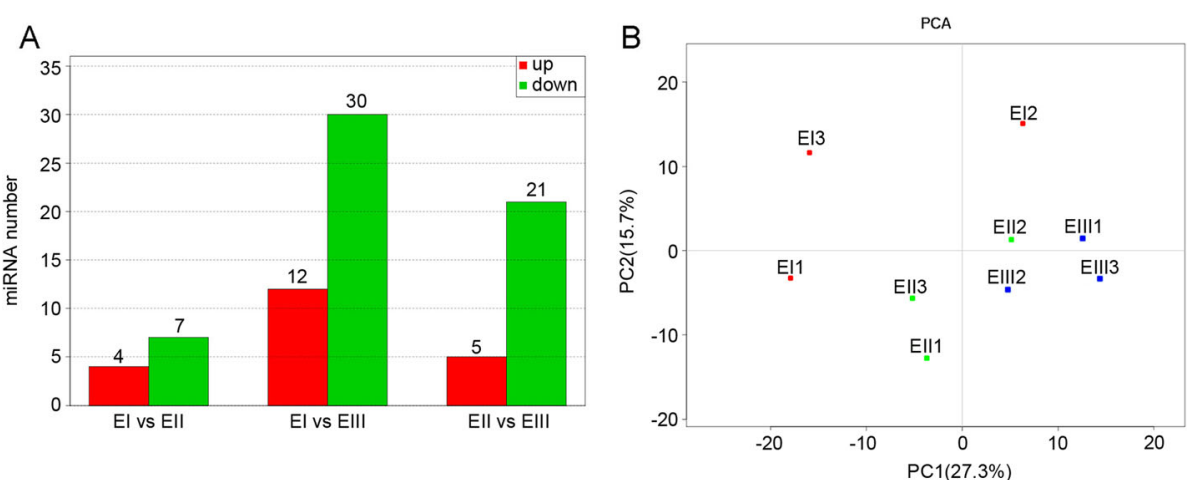

Fig. 5 Differential Expression of miRNAs in Different Stages of Internode Elongation in Sugarcane. a Number of differentially expressed miRNAs in the comparisons of EI vs. Ell, El vs. Elll, and Ell vs. Elll. Red bars represent the number of up-regulated miRNA. Green bars represent the number of down-regulated miRNAs. The number on each bar indicates the count of up-regulated or down-regulated miRNAs. b Two-component principal component analysis of the nine miRNA libraries from the pre-elongation stage (El), early elongation stage (EII) and rapid elongation stage (EllI). The percentages represent the variances captured by the principal component 1 (PC1) and principal component 2 (PC2). Red, blue and green dots showed El, Ell and EIII, respectively

pairs. Among all miRNA and mRNA pairs in "plant hormone signal transduction" pathways, 11 genes associated with auxin were found under regulation by miRNAs.

To confirm the results of small RNA-seq, qPCR was used to analyze miR2592-y, novel-m0204-5p, miR1520-x and miR6169-x. The expression profiles from $\mathrm{qPCR}$ were consistent with the results of the sequencing analysis (Fig. 7).

\section{Discussion}

Stem is the primary valuable tissue for sugarcane, which grows within the internode elongation process. To understand the miRNA-mRNA network during this important process, an integrated mRNA and small RNA sequencing study was done using a next-generation sequencing approach for different internode elongation stages in sugarcane in the present study. This pioneer study provided a

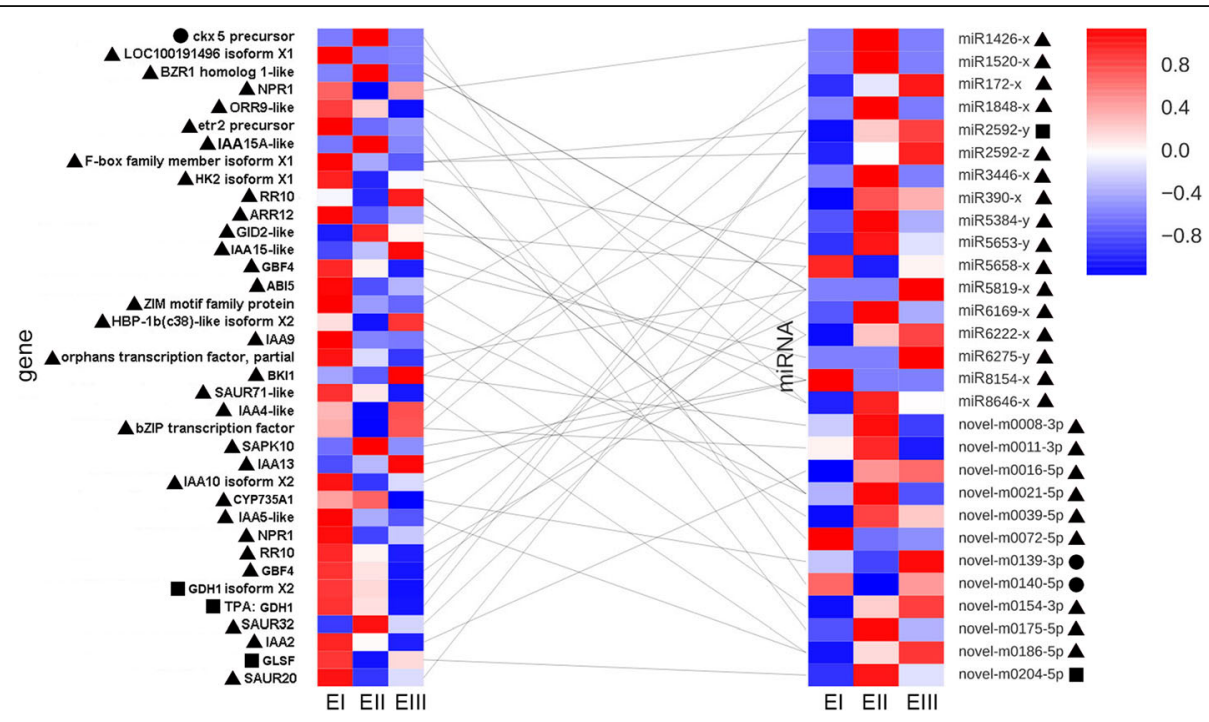

Fig. 6 Differentially Expressed miRNAs and their Targets in "Zeatin Biosynthesis", "Nitrogen Metabolism" and "Plant Hormone Signal Transduction" Pathways. Left: Heat map of miRNA targeted genes; Right: Heat map of the targets of miRNAs. Gene expression is colored for low (blue) to high (red) by e Z-score calculated from RPKM values. miRNA expression is colored for low (blue) to high (red) by e Z-score calculated from TPM values. The left panel: each line represents a library listed below and each row represents a single gene. The left panel: each line represents a library listed below and each row represents a single miRNA. El, Ell and EIII indicate Pre-elongation stage, elongation stage and rapid elongation stage, respectively. Circular, square and triangle indicate genes and miRNAs in "zeatin biosynthesis", "nitrogen metabolism" and "plant hormone signal transduction" pathways, respectively. Lines between gene and miRNA show the predicted interaction 

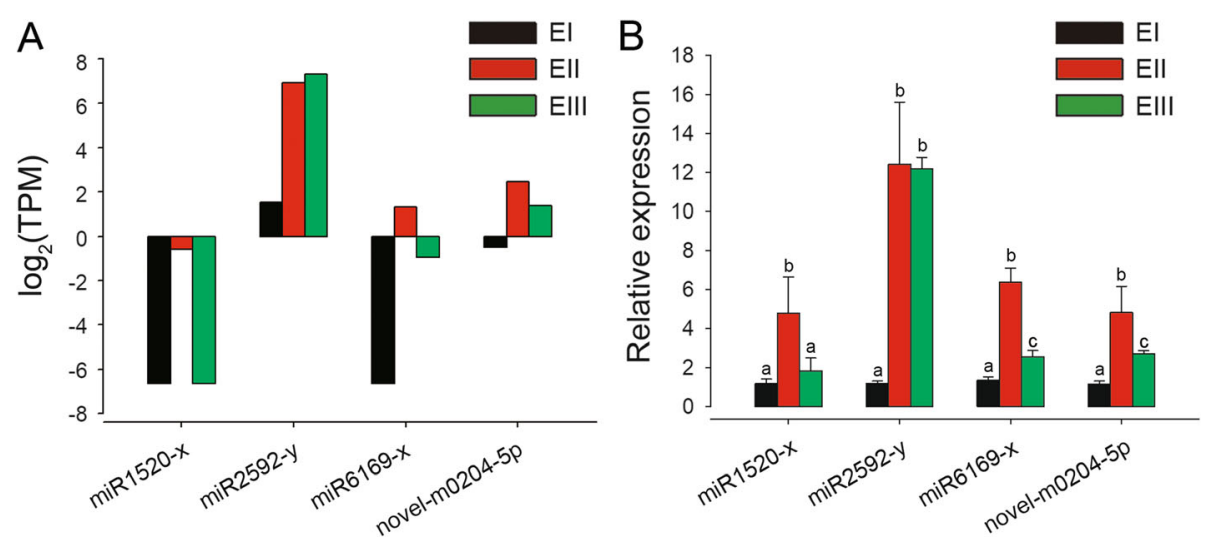

Fig. 7 Expression Levels of Four Candidate miRNAs in different stages. a Expression of four candidate miRNAs from RNA-seq calculated by the TPM method. The expression is shown as $\log _{2}$ (TPM). $\mathbf{b}$ Expression of four candidate miRNAs from qPCR. The qPCR results are presented at each stage as the mean $\pm S D$. The significant differences $(P<0.05)$ among the PCR results in different groups were indicated using different letters on each bar. El (black bars), Ell (red bars) and EIII (green bars) indicate pre-elongation stage, elongation stage and rapid elongation stage, respectively

molecular basis underlying the elongated internodes in posttranscription regulation.

To investigate the gene profiles in a wide range during sugarcane internode elongation, next-generation sequencing technology was used to analyze the transcriptome changes in the stems from the pre-elongation stage, early elongation stage and rapid elongation stage. The de novo assembly generated 80,745 unigenes. The number of differentially expressed genes in the EI vs. EII, EI vs. EIII and EII vs. EIII comparisons were 493, 5035 and 3041, respectively. Compared with the differentially expressed genes, a significantly higher ratio of unigenes showed similar expression among all the groups, strongly suggesting that the differentially expressed genes were involved in sugarcane internode elongation.

The enriched GO terms identified from the differentially expressed genes were within our expectations. Oxidoreductase activity and tetrapyrrole binding were highly enriched in EI compared with those in the other two groups, indicating the function of these genes in sugarcane internode elongation. Contributing to oxidoreductase activity, flavonoid 3-monooxygenase was significantly higher in the EI pre-elongation stage than in the other groups. Flavonoid 3-monooxygenase is responsible for catalyzing flavonoid to 3'-hydroxyflavonoid. Flavonoids affect plant resistance [42], and flavonoid accumulation may inhibit lignin synthesis [43]. Tetrapyrrole plays various roles in biological processes in plants, including plant growth [44]. For the tetrapyrrole binding term, higher expression of ent-kaurenoic acid oxidase 1 was identified at EII and EIII than EI. This enzyme catalyzes the gibberellin biosynthesis pathway and regulates lignin synthesis [45].

To understand the key pathways in sugarcane internode elongation, the enriched KEGG pathways with differentially expressed genes were identified, and "zeatin biosynthesis", "nitrogen metabolism" and "plant hormone signal transduction" pathways were enriched with differentially expressed genes. The differentially expressed genes in "zeatin biosynthesis" pathways included CKX3, CKX5, CKX9, CKX10 and CKO2 at EII and EIII compared with EI. It was noted that with the exception of CKX5 at EII, all the other CKX and CKO were significantly down-regulated at EII and EIII stages. CKX/CKO is responsible for degradation of cytokinin $[46,47]$. Low expression of the genes in these pathways might be related to high growth activities at EII and EIII stages during internode elongation via cytokinin accumulation [48]. Glutamate dehydrogenase and nitrate reductase were the two enzyme families in "nitrogen metabolism" pathways that might be involved in internode elongation. Transgenic studies in plants found that glutamate dehydrogenase is important for plant growth and productivity [49]. Nitrate as a nutrient for plant growth is regulated by nitrate reductase, indicating changes in nutrient requirements during sugarcane internode elongation [50]. The changing of "plant hormone signal transduction" during internode elongation was also expected. Auxin-related genes including auxin-responsive proteins were decreased at EII and EIII compared with those at EI. Auxin-responsive proteins affect auxin expression by acting on its promoter [51,52], leading to regulation of sugar accumulation, ethylene biosynthesis and promoting cell elongation.

The observations that several differentially expressed genes were related to internode elongation in the present study indicated that the sugarcane internode elongation was induced by the changes in gene expression in key pathways, suggesting a complex network of the relationships between hormone secretion and internode elongation in sugarcane.

Previous studies demonstrated that plant miRNAs regulate gene expression at posttranscriptional levels related to plant development, including germination, root 
development, flowering and internode elongation. Here, small RNA sequencing was performed in the stems from the pre-elongation stage, early elongation stage and rapid elongation stage in the present study. A total of 241 known miRNAs and 245 novel candidate miRNAs were identified in these small RNA libraries. Most of these miRNAs were expressed widely among the three different stages. miR168$x$, miR319-y, miR168-y, miR396-x and miR166-y were the most abundant known miRNAs, which were consistent with previous miRNA studies in sugarcane [53-55].

Subsequently, the miRNAs involved in control of the gene expression that was related to internode elongation was analyzed. As found in transcriptome analysis, several genes in "zeatin biosynthesis", "nitrogen metabolism" and "plant hormone signal transduction" pathways participated in sugarcane internode elongation. A total of 43 miRNA-mRNA pairs were found included. Only 6 miRNAs were decreased while other 37 miRNAs increased in EII and EIII stages. Thus, the overall expression trend of miRNAs in these pathways were downregulated and their targeted mRNA were upregulated during elongation process. Among them, CKX5 precursor and CYP735A1 were targeted by two novel miRNAs. This evidence showed that numbers of the candidate miRNA-mRNA remained unidentified to date. Further studies are required to demonstrate the substantive regulatory roles of the novel miRNAs. miR2592 is proposed to participate in the adventitious shoot organogenesis in Acacia crassicarpa [56]. In the present study, it was found that miR2592-y was up regulated at EIII and EII stages, whereas its targets were GDH1 isoform X2 and TPA, and GDH1 was down-regulated. In the "plant hormone signal transduction" pathway, 11 miRNAmRNA pairs were found related to auxin. In these pairs, 5 known miRNAs and 4 novel miRNAs were included. The 5 known miRNAs were miR1520-x, miR390-x, miR5658$\mathrm{x}$, miR6169-x and miR8154-x. miR1520 is unstable in sugarcane buds under cold stress [57] and only increased at EII stage, which would be expected to lead to an increase in repressing IAA4-like gene expression. The miR390 and its target as auxin response factor were previously reported [58], and therefore, the up-regulation at EII and EIII stages to affect the internode elongation was understandable. miR5658 targeted lateral suppressor-like protein (HaLS-L) and participated in growth and development, as reported previously in carrot [59]. Overexpression of miR8154 in Taxus cell lines indicates that miR8154 regulates Taxol, phenylpropanoid, and flavonoid biosynthesis pathways [60]. In this study, high expression of miR8154 at EI stage suggested that the activities of miR8154 were increasing at pre-elongation stage of internode in sugarcane.

With the integrated data from mRNA and small RNA sequencing at different stages of internode elongation, a comprehensive vision could be developed for understanding the miRNA function in this important biological process of sugarcane. Several differentially expressed genes involved in internode elongation related to "zeatin biosynthesis", "nitrogen metabolism" and "plant hormone signal transduction" pathways were identified. The miRNA data were then used to predict the target mRNA and putative mRNA-miRNA interactions that might participate in internode elongation were found (Fig. 8). Therefore, the present findings demonstrated that miRNAs mediated gene expression in sugarcane internode elongation, which indicates a regulatory network between miRNAs and genes at first glance.

\section{Conclusions}

In summary, mRNA and small RNA profiles were first revealed in sugarcane internode elongation, and comprehensive analysis was performed to identify the regulatory network during this biological process. The results suggested that potential miRNA-mRNA pairs involved in "zeatin biosynthesis", "nitrogen metabolism" and "plant hormone signal transduction" pathways controlled stem growth and development. This evidence provides valuable information for further functional characterization of the miRNAs and their targets in sugarcane internode elongation.

\section{Methods}

\section{Plant cultivation and tissue collection}

The sugarcane variety GT42, which was bred through sexual hybridization [61], was used as the material in the present study. The sugarcane was grown in an intelligent greenhouse at the Sugarcane Research Institute of Guangxi Academy of Agricultural Sciences, Nanning, China. The bud body from the upper middle stem was selected on March 15, 2016. The seedcane was cut into single-bud setts, and cultivated in a sand table. When the seedlings grew to a height of $8-10 \mathrm{~cm}$, those with consistent size were transplanted to an intelligent greenhouse. After two more month's growth, when 9-10 true leaves emerged, the plants entered the pre-elongation stage (EI), and the tissue of the stem internode covered by the second true leaf was collected. When 12-13 true leaves emerged, the plants entered the early elongation stage, and the tissue from the stem internode covered by the second true leaf was collected (EII). The rapid elongation stage was determined when 15-16 true leaves emerged, and the stem internode tissue covered by the second true leaf was collected (EIII). Three biological replicates (individuals) were included in the present study for each stage. These tissues were stored at $-80^{\circ} \mathrm{C}$ after liquid nitrogen treatment.

\section{Construction of RNA and small RNA sequencing libraries}

According to the supplier's instructions, total RNA was extracted with RNA Trizol (Invitrogen, Carlsbad, CA, 


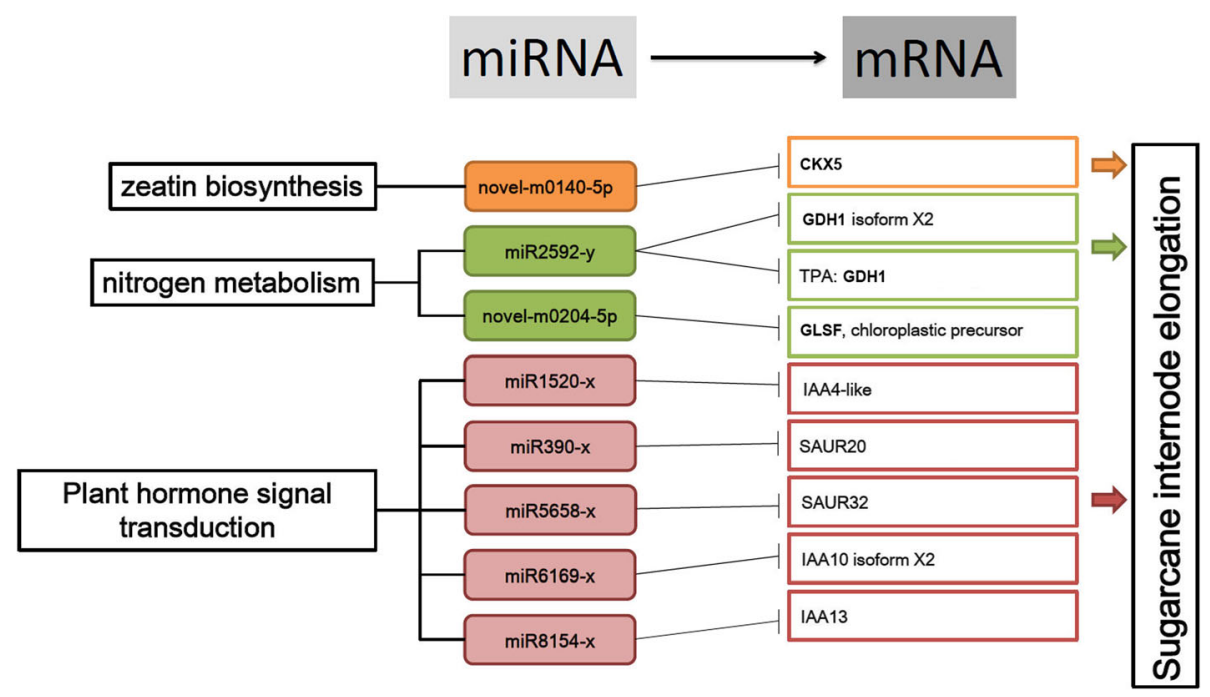

Fig. 8 A miRNA-Regulated mRNA Model in Sugarcane Internode Elongation. Novel-m0140-5p was predicted to regulate CKX5 in "zeatin biosynthesis". In "nitrogen metabolism" pathway, miR2592-y was predicted to regulate GDH1 isoform X2 and TPA: GDH1; novel-m0204-5p was predicted to target CLSF, chloroplastic precursor. miR1520-x, miR390-x, miR5658-x, miR6169-x, and miR8154-x were predicted to target IAA4-like, SAUR32, IAA10 isoform X2 and IAA13, respectively in "plant hormone signal transduction" pathway. These miRNAs targeted genes from "zeatin biosynthesis", "nitrogen metabolism" and "plant hormone signal transduction" pathways participate in sugarcane internode elongation

USA). The RNA integrity and quantity were detected by an Agilent 2100 bioanalyzer (Agilent, Santa Clara, CA, USA). Nine mRNA-seq libraries were constructed. First, the mRNAs were enriched by oligo $(\mathrm{dT})$ beads and fragmented into short fragments by fragmentation buffer; second, the first-strand cDNA was reversely transcribed by random primers followed by synthesis of the secondstrand cDNA using DNA polymerase I and RNase H; finally, the cDNA fragments were purified with a Qiaquick PCR extraction kit (Qiagen, Hilden, Germany) and ligated with Illumina sequencing adapters (Illumina, San Diego, CA, USA). The mRNA-seq libraries were prepared using pair-end methods (read length was 150 bp) and sequenced using a HiSeq ${ }^{\text {Tw }} 4000$ (Illumina, San Diego, CA, USA).

Small RNAs from total RNAs were isolated with 3.5\% agarose gel electrophoresis by isolating the 18-30 nt fragments. After ligation with $3^{\prime}$ adapters, the small RNAs were extracted by denaturing urea polyacrylamide gel electrophoresis by isolating the 36-44 nt desired band. Then, $5^{\prime}$ adapters were ligated with the isolated small RNAs following reverse transcription PCR, and the products were isolated again using 3.5\% agarose gel electrophoresis at $62-75 \mathrm{bp}$. The small RNA libraries were sequenced using a HiSeq 2000 (Illumina, San Diego, CA, USA).

Raw data processing and de novo assembly of mRNA-seq reads

Raw reads were stored as fastq and filtered to obtain high-quality clean reads. Briefly, the adapters from raw reads were first removed; then, the reads containing > $10 \%$ unknown nucleotides $(\mathrm{N})$ were excluded; and finally, low-quality reads that had 50\% low quality (qvalue lower than 10) bases were removed. The remaining reads were clean reads.

De novo assembly was conducted using the Trinity program (Version: v2.1.1). The inchworm assembled reads using a K-mer-based approach to generate contigs. Chryalis built a de Bruijn graph for clusters of contigs. Finally, based on de Bruijn graphs, Butterfly analyzed the read pairings from the contigs to generate transcripts and unigenes.

\section{Identification of differentially expressed genes}

Gene expression was calculated based on Reads Per kb per Million reads (RPKM) [62]. The RPKM formula was the following: $\mathrm{RPKM}=\left(1 \times 10^{6} \times \mathrm{C}\right) /(\mathrm{N} \times \mathrm{L} / 1000)$, where $\mathrm{C}$ represents the number of reads mapped to the target unigenes, $\mathrm{N}$ represents the number of reads mapped to all the unigenes, and $\mathrm{L}$ is the length of the target unigenes. The calculation was performed by the RSEM package. The adjustment of the $P$-value for multiple testing by FDR correction was performed for identification of differentially expressed genes with FDR cutoff $<$ 0.05 . The differentially expressed genes were identified using the standard as $\mid \log 2$ Ratio $\mid \geq 1$ and $\mathrm{q}<0.05$.

The unigenes from De novo assembly were annotated by four public databases, including the NCBI non-redundant protein database (www.ncbi.nlm.nih.gov), Swiss-Prot protein database (www.expasy.ch/sprot), KOG database (www. ncbi.nlm.nih.gov/KOG) and Kyoto Encyclopedia of Genes 
and Genomes database (www.genome.jp/kegg), using the Blastx package from NCBI (www.ncbi.nlm.nih.gov/ BLAST/). The cutoff e-value was 1e-5, and the bestaligned sequence was determined as the annotation for unigenes. Gene Ontology (GO) was used to reflect the gene function of the large unigene set. The GO annotation of the unigenes was performed using Blast2 $\mathrm{GO}$ software based on the annotation results from the NCBI non-redundant protein database.

\section{Processing of small RNA sequencing data}

The clean small RNA reads were obtained from raw reads after removing the adapters and low-quality reads (the reads with more than one base with a Q-value lower than 20). Only small RNAs ranging from 18 to $35 \mathrm{nt}$ in length were included in the further analysis. The clean small RNA reads were matched to the GenBank database (www.ncbi.nlm.nih.gov/genbank/, Version 209.0) and Rfam database (rfam.xfam.org/, Version 11) to identify and remove rRNA, scRNA, snRNA and tRNA by blastn (the cutoff e-value was 1e-5).

\section{Identification of known miRNAs and novel miRNAs}

Known miRNAs were identified by hitting the candidate miRNAs miRBase (www.mirbase.org/, Version 21) using blastn (the cutoff e-value was 1e-5). The annotated miRNAs were determined as known miRNAs. Novel miRNAs were predicted by mireap v0.2 (https://sourceforge. net/projects/mireap/). The parameters of mireap were -A 18, -B 25, -a 20, -b 23, - u 20, -e 18, -d 300, -p 16, $-\mathrm{v} 4,-\mathrm{s} 4$, and $-\mathrm{f} 20$.

\section{Prediction of the targets of miRNAs}

The software patmatch (ftp://ftp.arabidopsis.org/home/ tair/Software/Patmatch/, Version1.2) with default parameters was used to predict the targets of miRNAs. Then, based on the patmatch prediction, the genemiRNA pairs with Spearman's correlation coefficient for ranked data lower than -0.5 were further confirmed the targets of miRNAs. The target gene functions of miRNAs were clarified by GO and KEGG annotation. GO enrichment was calculated by FDR correction, with FDR lower than 0.05 as significantly enriched. The KEGG pathways were performed with FDR correction, with FDR lower than 0.05 as a threshold.

\section{Identification of differentially expressed miRNAs}

The expression of miRNAs was assessed by transcripts per million $(\mathrm{TPM})$, and $\mathrm{TPM}=$ miRNA counts $/($ Total miRNA counts $\times 10^{6}$ ). The fold-changes were calculated using the formula fold-changes $=\log 2$ (group1/group2). The miRNAs with fold change greater than 2 and $P$ value less than 0.05 were identified as differentially expressed miRNAs.

\section{Quantitative PCR (qPCR)}

Sugarcane tissues were collected as described previously. The total RNA from the different stages EI, EII and EIII was extracted with RNA Trizol (Invitrogen, Carlsbad, CA, USA). Ten of the differentially expressed genes from "zeatin biosynthesis", "nitrogen metabolism" and "plant hormone signal transduction" pathways were selected for confirmation by qRT-PCR. The primers for qPCR were designed by Primer Express v3.0 (Applied Biosystems, Waltham, MA, USA) (Additional file 8). $\beta$-actin was used as the internal control. After RNA extraction, the first-strand cDNA synthesis was obtained using total RNA and a PrimeScript RT Reagent Kit with gDNA Eraser (TaKaRa, Dalian, China) following the manufacturer's instructions. The cDNA was diluted to 1:20 and used as template for qPCR, which was performed using SYBR $^{\circ}$ Premix Ex Taq ${ }^{\text {Tix }}$ II (TliRNaseH Plus) (TakaRa, Dalian, China) according to the manufacturer's instructions. The PCR was started with initial denaturation at $95^{\circ} \mathrm{C}$ for $30 \mathrm{~s}$, followed by 40 amplification cycles at $95^{\circ} \mathrm{C}$ for $10 \mathrm{~s}$ and $60^{\circ} \mathrm{C}$ for $30 \mathrm{~s}$ using an ABI 7500 Fast Real Time PCR System (Applied Biosystems, Waltham, MA, USA). The threshold cycle (CT) values were used to calculate relative expression by the $2^{-\Delta \Delta C T}$ method [63], which was performed using 7500 software version 2.0.4 (Applied Biosystems, Waltham, MA, USA). For qPCR of miRNA, the reverse transcriptase reaction was performed using a microRNA RT kit (TakaRa, Dalian, China). The cycles were the same as with mRNA analysis. All the reactions were performed with three biological repeats. The data were presented as the mean \pm $\mathrm{SD}$. The significant differences among the groups were assessed using one-factor analysis of variance (one-way ANOVA) followed by a Bonferroni post hoc test. Pvalue $<0.05$ was considered statistically significant.

\section{Supplementary information}

Supplementary information accompanies this paper at https://doi.org/10. 1186/s12864-019-6201-4.

\footnotetext{
Additional file 1. The RPKM of genes by RNA-Seq.

Additional file 2. $\mathrm{GO}$ analysis of differentially expressed genes.

Additional file 3. KEGG pathway of differentially expressed genes.

Additional file 4. Novel miRNAs predicted by mireap $v 0.2$.

Additional file 5. miRNA TPM values.

Additional file 6. miRNA target predictions.

Additional file 7. The differentially expressed mRNA and miRNA pairs in "zeatin biosynthesis", "nitrogen metabolism" and "plant hormone signal transduction" pathways.

Additional file 8. Primers used in this study.
}

\section{Abbreviations}

CKX5: Cytokinin dehydrogenase 5 precursor; $C T$ : Threshold cycle;

CYP735A1: Cytokinin hydroxylase-like isoform X1; El: Pre-elongation stage; Ell: Early elongation stage; Elll: Rapid elongation stage; FDR: False discovery rate; GDH1: Glutamate dehydrogenase; GLSF: Ferredoxin-dependent 
glutamate synthase; GO: Gene Ontology; HaLS-L: Lateral suppressor-like protein; KEGG: Kyoto encyclopedia of genes and genomegenomes; kOG: Eukaryotic orthologous group; miRNA: MicroRNA; qPCR: Quantitative $P C R ;$ RNA-seq: RNA sequencing or the transcriptome sequencing; RPKM: Reads Per kb per Million reads; rRNA: Ribosomal ribonucleic acid SD: Standard deviation; snoRNA: Small nucleolar RNA; snRNA: Small nuclear ribonucleic acid; TPM: Transcripts per million; tRNA: Transfer RNA

\section{Acknowledgements}

The authors are grateful to Dr. Prakash Lakshmanan (E-Mail: plakshmanan201 8@outlook.com) for his critical revision of this manuscript. We thank Pu Li from Guangxi Pfomic Info Technology Co., Ltd. for the technical support of bioinformatics analysis.

\section{Authors' contributions}

$L Q, R C$, and $Y F$ performed the experiments. $X H$ and $H L$ collected materials. $F X, J L 1$ and RZ analyzed the data. LQ, JL2, HZ, JW and YL wrote the paper. All authors participated in the discussion and read and approved the final manuscript.

\section{Funding}

National Natural Science Foundation of China (31701363 and 31360312), Guangxi Natural Science Foundation Project (2018GXNSFAA138149, 2017GXNSFBA198050, 2016GXNSFBA380034, 2015GXNSFDA139011 and 2015GXNSFBA139095), Guangxi Key Laboratory Construction Project (15-14013 and 16-380-18), Guangxi Science and Technology Project (Guike AA17202042-13), Guangxi Bangui Scholars and Special Experts Special Funds (2013), Guangxi Academy of Agricultural Sciences Fund Project (2015YM13 2018YM02 and 2018YT01), and National Modern Agricultural History Technology System, Guangxi Sugarcane Innovation Team Project (nycytxgxcxtd-03-01). The funding bodies had no role in study design, data collection and analysis, decision to publish, or preparation of the manuscript.

\section{Availability of data and materials}

All the raw data are available in the Sequencing Read Archive (SRA) of NCBI under the BioProject number PRJNA429786.

\section{Ethics approval and consent to participate}

No special permits were required to collect tissues from sugarcane.

\section{Consent for publication}

Not applicable.

\section{Competing interests}

The authors declare that they have no competing interests.

\section{Author details}

'Sugarcane Research Institute, Guangxi Academy of Agricultural Sciences/ Sugarcane Research Center, Chinese Academy of Agricultural Sciences, East Daxue Road 172, Nanning 530004, Guangxi, China. ${ }^{2}$ Key Laboratory of Sugarcane Biotechnology and Genetic Improvement (Guangxi), Ministry of Agriculture, and Guangxi Key Laboratory of Sugarcane Genetic Improvement, Nanning, Guangxi, China.
\end{abstract}

\section{Received: 2 October 2018 Accepted: 18 October 2019} Published online: 07 November 2019

\section{References}

1. Lingle SE, Thomson JL. Sugarcane internode composition during crop development. Bioenergy Res. 2012;5:168-78.

2. Li J, Sima W, Ouyang B, Wang T, Ziaf K, Luo Z, et al. Tomato SIDREB gene restricts leaf expansion and internode elongation by downregulating key genes for gibberellin biosynthesis. J Exp Bot. 2012;63:6407-20.

3. Zhou H-L, He S-J, Cao Y-R, Chen T, Du B-X, Chu C-C, et al. OsGLU1, a putative membrane-bound endo-1,4-B-D-glucanase from rice, affects plant internode elongation. Plant Mol Biol. 2006;60:137-51.

4. Cui K, He C-Y, Zhang J-G, Duan A-G, Zeng Y-F. Temporal and spatial profiling of internode elongation-associated protein expression in rapidly growing culms of bamboo. J Proteome Res. 2012;11:2492-507.
5. Zhao K, Tung C-W, Eizenga GC, Wright MH, Ali ML, Price AH, et al. Genomewide association mapping reveals a rich genetic architecture of complex traits in Oryza sativa. Nat Commun. 2011;2:467.

6. Sripongpangkul K, Posa GBT, Senadhira DW, Brar D, Huang N, Khush GS, et al. Genes/QTLs affecting flood tolerance in rice. Theor Appl Genet. 2000; 101:1074-81.

7. Bishop GJ, Koncz C. Brassinosteroids and plant steroid hormone signaling. Plant Cell. 2002;14:S97-S110.

8. Hager A. Role of the plasma membrane $\mathrm{H}+$ +-ATPase in auxin-induced elongation growth: historical and new aspects. J Plant Res. 2003;1 16:483505.

9. La Nafie YA, Santos CBD, Brun FG, Van Katwijk MM, Bouma TJ. Waves and high nutrient loads jointly decrease survival and separately affect morphological and biomechanical properties in the seagrass Zostera noltii. Limnol Oceanogr. 2012;57:1664-72.

10. Hwang SJ, Hamayun M, Kim HY, Na Cl, Kim KU, Shin DH, et al. Effect of nitrogen and silicon nutrition on bioactive gibberellin and growth of rice under field conditions. J Crop Sci Biotechnol. 2008;10:281-6.

11. Peralta G, Pérez-Lloréns JL, Hernández I, Vergara JJ. Effects of light availability on growth, architecture and nutrient content of the seagrass Zostera noltii Hornem. J Exp Mar Biol Ecol. 2002;269:9-26.

12. Hattori Y, Miura K, Asano K, Yamamoto E, Mori H, Kitano H, et al. A major QTL confers rapid internode elongation in response to water rise in Deepwater rice. Breed Sci. 2007;57:305-14.

13. Mazzella MA, Bertero D, Casal JJ. Temperature-dependent internode elongation in vegetative plants of Arabidopsis thaliana lacking phytochrome B and cryptochrome 1. Planta. 2000;210:497-501.

14. Cleland RE. Auxin and cell elongation. In: Davies PJ, editor. Plant hormones: biosynthesis, signal transduction, action! London: Springer; 2010. p. 204-20.

15. Davies PJ. The plant hormones: their nature, occurrence, and function. In: Davies PJ, editor. Plant hormones: biosynthesis, signal transduction, action! Dordrecht: Springer; 2010. p. 1-15.

16. Davies PJ. Plant hormones and their role in plant growth and development. Berlin/Heidelberg: Springer Science \& Business Media Press; 2012

17. Ingram TJ, Reid JB, MacMillan J. The quantitative relationship between gibberellin A1 and internode growth in Pisum sativum L. Planta. 1986;168:414-20.

18. Clouse SD, Sasse JM. BRASSINOSTEROIDS: essential regulators of plant growth and development. Annu Rev Plant Physiol Plant Mol Biol. 1998;49: 427-51.

19. Azuma T, Hirano T, Deki Y, Uchida N, Yasuda T, Yamaguchi T. Involvement of the decrease in levels of abscisic acid in the internodal elongation of submerged floating rice. J Plant Physiol. 1995;146:323-8.

20. Metraux JP, Kende $\mathrm{H}$. The role of ethylene in the growth response of submerged deep water rice. Plant Physiol. 1983;72:441-6.

21. Heinrich M, Hettenhausen C, Lange T, Wünsche H, Fang J, Baldwin IT, et al. High levels of jasmonic acid antagonize the biosynthesis of gibberellins and inhibit the growth of Nicotiana attenuatastems. Plant J. 2013;73:591-606.

22. Pieterse CMJ, Van der Does D, Zamioudis C, Leon-Reyes A, Van Wees SCM. Hormonal modulation of plant immunity. Annu Rev Cell Dev Biol. 2012;28: 489-521.

23. Costantini E, Landi L, Silvestroni O, Pandolfini T, Spena A, Mezzetti B. Auxin synthesis-encoding transgene enhances grape fecundity. Plant Physiol. 2007;143:1689-94.

24. Schijlen EGWM, de Vos CHR, Martens S, Jonker HH, Rosin FM, Molthoff JW, et al. RNA interference silencing of chalcone synthase, the first step in the flavonoid biosynthesis pathway, leads to parthenocarpic tomato fruits. Plant Physiol. 2007;144:1520-30.

25. Wasson AP, Pellerone FI, Mathesius U. Silencing the flavonoid pathway in Medicago truncatula inhibits root nodule formation and prevents auxin transport regulation by rhizobia. Plant Cell. 2006;18:1617-29.

26. Denance N, Sanchez-Vallet A, Goffner D, Molina A. Disease resistance or growth: the role of plant hormones in balancing immune responses and fitness costs. Front Plant Sci. 2013;4:155

27. Guo HS, Xie Q, Fei J-F, Chua N-H. MicroRNA directs mRNA cleavage of the transcription factor NAC1 to downregulate auxin signals for Arabidopsis lateral root development. Plant Cell. 2005;17:1376-86.

28. Jones-Rhoades MW, Bartel DP. Computational identification of plant microRNAs and their targets, including a stress-induced miRNA. Mol Cell. 2004; 14:787-99.

29. Si-Ammour A, Windels D, Arn-Bouldoires E, Kutter C, Ailhas J, Meins F, et al. miR393 and secondary siRNAs regulate expression of the TIR1/AFB2 auxin 
receptor clade and auxin-related development of Arabidopsis leaves. Plant Physiol. 2011;157:683-91.

30. Tsuji H, Aya K, Ueguchi-Tanaka M, Shimada Y, Nakazono M, Watanabe R, et al. GAMYB controls different sets of genes and is differentially regulated by microRNA in aleurone cells and anthers. Plant J. 2006:47:427-44.

31. Cheng HY, Wang $Y$, Tao X, Fan YF, Dai $Y$, Yang H, et al. Genomic profiling of exogenous abscisic acid-responsive microRNAs in tomato (Solanum lycopersicum). BMC Genomics. 2016;17:423.

32. Edwards D, Batley J. Plant genome sequencing: applications for crop improvement. Plant Biotechnol J. 2010:8:2-9.

33. Varshney RK, Nayak SN, May GD, Jackson SA. Next-generation sequencing technologies and their implications for crop genetics and breeding. Trends Biotechnol. 2009;27:522-30.

34. Wang Z, Gerstein M, Snyder M. RNA-Seq: a revolutionary tool for transcriptomics. Nat Rev Genet. 2009;10:57-63.

35. Cui K, Wang H, Liao S, Tang Q, Li L, Cui Y, et al. Transcriptome sequencing and analysis for culm elongation of the world's largest bamboo (Dendrocalamus sinicus). PLoS One. 2016;11:e0157362.

36. Wei X, Zhang W, Zhang Q, Sun P, Li Z, Zhang M, et al. Analysis of differential expression of genes induced by ethephon in elongating internodes of maize plants. Front Agric Sci Eng. 2016;3:263-82.

37. Wang Y, Ding Y, Liu J-Y. Identification and profiling of microRNAs expressed in elongating cotton fibers using small RNA deep sequencing. Front Plant Sci. 2016;7:1722.

38. Tew TL, Cobill RM. Genetic improvement of sugarcane (Saccharum spp.) as an energy crop. In: Vermerris W, editor. Genetic improvement of bioenergy crops. New York: Springer; 2008. p. 273-94.

39. Nonato RV, Mantelatto PE, Rossell CE. Integrated production of biodegradable plastic, sugar and ethanol. Appl Microbiol Biotechnol. 2001; 57:1-5.

40. Peng Z, Zhang C, Zhang Y, Hu T, Mu S, Li X, et al. Transcriptome sequencing and analysis of the fast growing shoots of moso bamboo (Phyllostachys edulis). PLoS One. 2013;8:e78944.

41. Bosch M, Mayer C-D, Cookson A, Donnison IS. Identification of genes involved in cell wall biogenesis in grasses by differential gene expression profiling of elongating and non-elongating maize internodes. J Exp Bot. 2011;62:3545-61

42. Havsteen BH. The biochemistry and medical significance of the flavonoids. Pharmacol Ther. 2002;96:67-202.

43. Besseau S, Hoffmann L, Geoffroy P, Lapierre C, Pollet B, Legrand M. Flavonoid accumulation in Arabidopsis repressed in lignin synthesis affects auxin transport and plant growth. Plant Cell. 2007;19:148-62.

44. Tanaka R, Tanaka A. Tetrapyrrole biosynthesis in higher plants. Annu Rev Plant Biol. 2007;58:321-46.

45. Helliwell CA, Chandler PM, Poole A, Dennis ES, Peacock WJ. The CYP88A cytochrome P450, ent-kaurenoic acid oxidase, catalyzes three steps of the gibberellin biosynthesis pathway. Proc Natl Acad Sci U S A. 2001;98:2065-70.

46. Schmülling T, Werner T, Riefler M, Krupková E, Manns IB. Structure and function of cytokinin oxidase/dehydrogenase genes of maize, rice, Arabidopsis and other species. J Plant Res. 2003;116:241-52.

47. Frebort I, Kowalska M, Hluska T, Frebortova J, Galuszka P. Evolution of cytokinin biosynthesis and degradation. J Exp Bot. 2011;62:2431-52.

48. Caboni E, D'Angeli S, Chiappetta A, Innocenti AM, van Onckelen H, Damiano C. Adventitious shoot regeneration from vegetative shoot apices in pear and putative role of cytokinin accumulation in the morphogenetic process. Plant Cell Tissue Organ Cult. 2002;70:199-206.

49. Dubois F, Tercé-Laforgue T, Gonzalez-Moro M-B, Estavillo J-M, Sangwan R, Gallais A, et al. Glutamate dehydrogenase in plants: is there a new story for an old enzyme? Plant Physiol Biochem. 2003;41:565-76.

50. Crawford NM. Nitrate: nutrient and signal for plant growth. Plant Cell. 1995; 7:859-68.

51. Hagen G, Guilfoyle T. Auxin-responsive gene expression: genes, promoters and regulatory factors. Plant Mol Biol. 2002;49:373-85.

52. Tiwari SB, Hagen G, Guilfoyle T. The roles of auxin response factor domains in auxin-responsive transcription. Plant Cell. 2003;15:533-43.

53. Bottino MC, Rosario S, Grativol C, Thiebaut F, Rojas CA, Farrineli L, et al. High-throughput sequencing of small RNA transcriptome reveals salt stress regulated microRNAs in sugarcane. PLoS One. 2013;8:e59423.

54. Gentile A, Ferreira TH, Mattos RS, Dias LI, Hoshino AA, Carneiro MS, et al. Effects of drought on the microtranscriptome of field-grown sugarcane plants. Planta. 2013;237:783-98.
55. Ortiz-Morea FA, Vicentini R, Silva GFF, Silva EM, Carrer H, Rodrigues AP, et al. Global analysis of the sugarcane microtranscriptome reveals a unique composition of small RNAs associated with axillary bud outgrowth. J Exp Bot. 2013;64:2307-20.

56. Liu W, Yu W, Hou L, Wang X, Zheng F, Wang W, et al. Analysis of miRNAs and their targets during adventitious shoot organogenesis of Acacia crassicarpa. PLoS One. 2014;9:e93438

57. Yang $Y$, Zhang $X$, Chen $Y$, Guo J, Ling H, Gao S, et al. Selection of reference genes for normalization of microRNA expression by RT-qPCR in sugarcane buds under cold stress. Front Plant Sci. 2016;7:86.

58. Marin E, Jouannet V, Herz A, Lokerse AS, Weijers D, Vaucheret H, et al. miR390, Arabidopsis TAS3 tasiRNAs, and their auxin response factor targets define an autoregulatory network quantitatively regulating lateral root growth. Plant Cell. 2010;22:1104-17.

59. Sarangzai AM. Profiling the carrot (Daucus carota L.) microRNAs and their targets. Pak J Bot. 2013;45:353-8.

60. Zhang M, Dong Y, Nie L, Lu M, Fu C, Yu L. High-throughput sequencing reveals miRNA effects on the primary and secondary production properties in long-term subcultured Taxus cells. Front Plant Sci. 2015;6:604.

61. Wang LW, Liao JX, Tan F, Tang SY, Huang JY, Li X, et al. Breeding of new high-yield, high-sugar and lodging-resistant sugarcane variety Guitang 42 and its high-yield cultivation technique. J Southern Agric. 2015;46:1361-6.

62. Mortazavi A, Williams BA, McCue K, Schaeffer L, Wold B. Mapping and quantifying mammalian transcriptomes by RNA-Seq. Nat Methods. 2008; 5:621-8.

63. Livak KJ, Schmittgen TD. Analysis of relative gene expression data using real-time quantitative $P C R$ and the $2-\Delta \Delta C T$ method. Methods. 2001;25: $402-8$.

\section{Publisher's Note}

Springer Nature remains neutral with regard to jurisdictional claims in published maps and institutional affiliations.

Ready to submit your research? Choose BMC and benefit from:

- fast, convenient online submission

- thorough peer review by experienced researchers in your field

- rapid publication on acceptance

- support for research data, including large and complex data types

- gold Open Access which fosters wider collaboration and increased citations

- maximum visibility for your research: over $100 \mathrm{M}$ website views per year

At $\mathrm{BMC}$, research is always in progress.

Learn more biomedcentral.com/submissions 